MATEC Web of Conferences 44, 02089 (2016)

DOI: $10.1051 /$ matecconf/20164402089

(C) Owned by the authors, published by EDP Sciences, 2016

\title{
A Qos Routing protocol based on AODV
}

\author{
Yong Qiang $\mathrm{Li}^{1}$, Yun Jie Zhu ${ }^{1}$, Qing Gang Fan ${ }^{1}$, Yu Bin $W u^{1}$ \\ ${ }^{1}$ Computer Staff Room, Xian Research Institute of High Technology, Xian 710025, China
}

\begin{abstract}
A Qos Routing protocol based on AODV (QOSAODV) is proposed aiming at the problem of the traditional QoS routing protocol which can not be applied perfectly in VANET. The weakness of the AODV routing protocol mechanism and the protocol itself are represented. By the simulation, the effectiveness and feasibility were completely verified. QOSAODV shows the superior performances in effectively lowering the routing broke ratio as well as packet drop ratio significantly, meanwhile, increasing the network throughput and scale.
\end{abstract}

Key Words. VANET,QoS, AODV,QOSAODV,NS2, routing broke ratio

\section{1 introduction}

At present ,Routing protocol for vehicular ad hoc networks[1] has being hotspot at home and abroad. in order to improve its performance, many routing protocols has been proposed. Most of them concentrated on the use of geographic information system (GIS) vehicle routing protocol research. However, the routing protocol algorithm based on geographical location information complexity is high, the node real-time computation load is bigger, and abnormal GPS devices, protocol cannot work at all. And in terms of QoS[2]security, almost follows the traditional QoS Ad Hoc network routing protocol mechanism, unable to reflect the characteristics of vehicular ad hoc networks. Based on the above situation, this paper proposes is not based on the geographic location information of the o vehicular ad hoc networks routing protocol strategy, introducing QoS safeguard mechanism of AODV routing protocol, and make it adapted to the vehicle mobile environment.

In the AODV protocol[3], each node maintains a neighbor list inside, which only save IP address and sequence number of the neighbor, so data forwarding do not know and the link between the neighbor node which is more suitable for data forwarding, and blind selection and forwarding. But the actual link is much different from the ideal situation, especially in the road between the link status of the vehicle is more obvious. Because of its direction are different for several vehicles in the one hop range, so it can maintain effective communication mobile distance difference is great, which shows do not consider the link state and random neighbor selection as forwarding nodes of AODV strategy will often lead to link the frequent fracture.

If the node can know the state of the link between itself and the neighbor node in real time, it can effectively carry out the selective forwarding, which can improve the effective maintenance time of the link. And in single frequency wireless channel environment, each node are and the neighbor nodes share the limited wireless bandwidth resources, so through the method of monitoring the idle channel, channel access can use the degree of bandwidth[4], can be in accordance with the task transmission bandwidth requires the selection of bandwidth wider links. In this way, it can improve the performance of the AODV protocol, and also can achieve the classification of QoS tasks. Here is a reflection of this idea, a QoS strategy is proposed: (1) when the source node sends RREQ to control the message, the bandwidth requirement of the task and the link stability are written into RREQ and broadcast. (2)when the intermediate

This is an Open Access article distributed under the terms of the Creative Commons Attribution License 4.0, which permits unrestricted use distribution, and reproduction in any medium, provided the original work is properly cited. 
nodes receive the RREQ packet, it first to compare their bandwidth ability and RREQ packet demands the bandwidth of the value, if not satisfied without forwarding, it discarded directly. If meet, then compare the previous radio RREQ neighbor nodes and their link-state values and RREQ packet request link stability value, if meet the forwarding, if does not meet the discarded directly, not to deal with. (3) when the destination node receives RREQ packet, they also compare their bandwidth capacity and link stability. by comparison, they embed the minimum RREP message and reply to the source node. (4) when the source node received the corresponding RREP message ,they extract its bandwidth value and stability of the link, update the routing tables, and data forwarding.

This QoS routing forwarding mechanism can effectively avoid the lack of poor condition of node and poor bandwidth link, choose a more stable and sufficient bandwidth of the link. But also some deficiencies, for example, if the intermediate node through the comparison, they will lost RREQ packet, they unable to find a satisfactory routing and lead to the routing request packet radio invalid, consumption of network bandwidth[5]. Also, if the source node find the routing path of failure for the first time, because of the change of the unpredictable link-state, the link disconnected, until received RRER message to re-launch the routing request ,they operation. Through repeated experiment and simulation, the mechanism does not significantly improve performance of routing vehicular ad hoc networks protocol.

How to accurately obtain the node itself available bandwidth capacity and link between neighbor nodes is the key of the routing protocol design performance, which influence QoS mechanism of state . Due to considering the portability of mobile terminals and CPU capacity, extract QoS index and the index of compare and select the operation and routing algorithm is not too complicated. This article take the simple and effective mathematical expectation and mathematical statistical methods to calculate the variance QoS parameter values.

(1) the available bandwidth values for:

When each node needs to know its own bandwidth available, it will access the MAC layer, and obtained value of the available bandwidth by the method of mathematical expectation. The formula is as follows:

Bandwidth $=\frac{1}{n} \sum_{i=1}^{n} \operatorname{Isidle}(i)$,

$1 \geq$ Bandwidth $\geq 0$

(2) Link stability degree of access:

Link_Stability $=\frac{1}{m} \sum_{i=1}^{m}\left(\Delta T_{i}-\sum \Delta T_{i}\right)^{2}$

In formula, $\Delta \mathrm{T}_{\mathrm{i}}$ As type of HELLO message of twice arrival interval time. In this paper, $\mathrm{m}$ was set to 10 , Link Stability set to 100 ; if the calculation result more than this value, it is set to $100.10 \geq$ Stability_Link $\geq 0$,if the interval of the HELLO information received are the same, it is very ideal link-state; stability degree is 0 , for the best. Actual comparing with the inside of the RREQ packet link stability degree, they need a conversion function, depending on the environment Settings.

\section{The QOSAODV Protocol}

QOSAODV routing protocol divided into the following three steps:

(1) routing request initiated: When node receives from the upper after sending a data packet, they first check for efficient reach the destination node routing. if there are, the data packet are direct forwarded; if there are not, request is routed. Before the data was cache, message protocol extract the data packet header QoS mission requirements to carry value, and record in the routing table. Protocol use the extracted Qo S demand value do a RREQ packets and send to the neighbor node, in the form of broadcast at the same time open expansion in search and TTL increase mechanism. After waiting for a period of time, if the node have not received the corresponding RREP message, then it resend RREQ packets, if it still no response after several attempts, think this node is inaccessible, and this node sending RREQ operation is prohibited within a period of time. (2) request of routing forwarding: After intermediate node received RREQ message ,the node view check RREQ packet that have received the same source node in the recent period of time, if there are, they give up directly, if not they cache the RREQ packet and compare Qo S index. Node obtain its own bandwidth available capacity value and Link 
stability value that node forward the RREQ[6] packet between a node and myself, and compare with value of QoS of RREQ packet required .

Min_Bandwidth $=\min \left(B W \_\right.$node ,BW_rreq) BW_node as the available bandwidth value of the node itself, BW_rreq RREQ packet for the specified minimum bandwidth requirements.

Min_LinkStability $=\min \left(L S \_n o d e \leftrightarrow p r e n o d e, L S \_r r e q\right)$

LS_node $\leftrightarrow$ prenode is degree of stability between the node itself with neighbor node, LS_rreq RREQ is stability degree value, which were specified for packet. Intermediate nodes update routing table (create a reverse routing, record the minimum in routing table) with the minimum, and the minimum cover RREQ packet QoS value, forward. This mechanism ensure that the QoS value of RREQ packet records is always the optimal bandwidth value and best link stability.

(3) respond routing: Receiving RREQ packets, destination node, as well as the operation of the intermediate nodes, update the routing table, and concluded minimum value of QoS. In order to respond to the source node, destination node make a RREP message, QoS minimum value are written into the RREP message of destination node, and sent to the source node. RREP reply according to the RREQ packet forwarding generated in the process of reverse routing, the source node to unicast.

\section{Simulation}

This article uses the Manhattan model[7], The specific parameters of the simulation environment such as the following Table3-1. The proposed QOSAODV protocol's performance is analyzed using NS2 simulator.

Table 3.1 The results of simulation analysis

\begin{tabular}{|c|c|}
\hline application layer & CBR \\
\hline Transport Laye & UDP \\
\hline Routing Layer & $\begin{array}{c}\text { QOSADOV 、 } \\
\text { AODV }\end{array}$ \\
\hline MAC Layer & 802.11DCF \\
\hline Wireless transmission model & $\begin{array}{c}\text { shadowing mode } \\
\beta=2.4\end{array}$ \\
\hline Wireless transmission frequency & $2.4 \mathrm{GH}$ \\
\hline Number of nodes & $\begin{array}{c}20,40,60,80, \\
100,120,140, \\
160,180,200\end{array}$ \\
\hline Scene size & $1000 \mathrm{~m} \times 1000 \mathrm{~m}$ \\
\hline simulation time & $100 \mathrm{~s}$. \\
\hline
\end{tabular}

Packet Drop Ratio

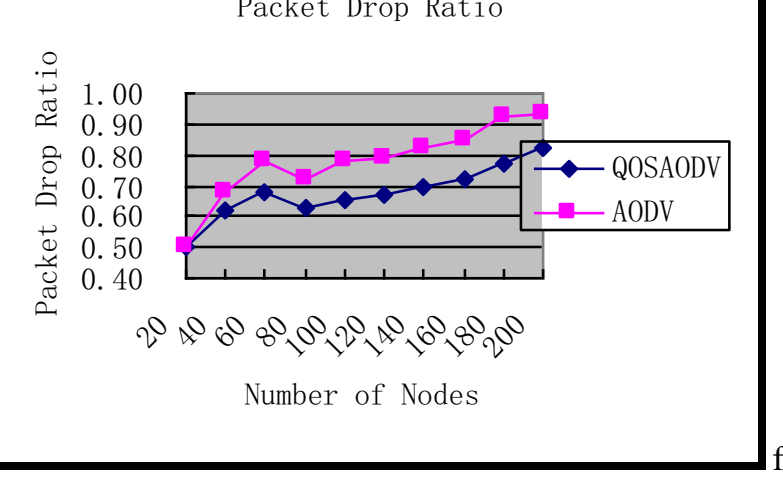

Figure 3.1 The comparison of packet loss rate

As you can see from figure 3.1, QOSAODV routing protocol AODV routing protocol and because of the increase of the number of nodes, the packet loss rate also will increase. But QOSAODV routing protocols due to its QoS safeguard mechanism, not because the number of nodes increases with the increase of packet loss rate, the number of nodes is long can reduce the packet loss rate of $11 \%$ on average, the node number once due to reach the destination node can be found with path is limited, so the two protocols performance are similar, but the number of nodes for QOSAODV routing protocol can effectively avoid the link to bedevil node and choose the forward routing path as the data is more stable, can effectively restrain the increase of the packet loss rate, intuitive proved the feasibility and effectiveness of the QOSAODV routing protocols.

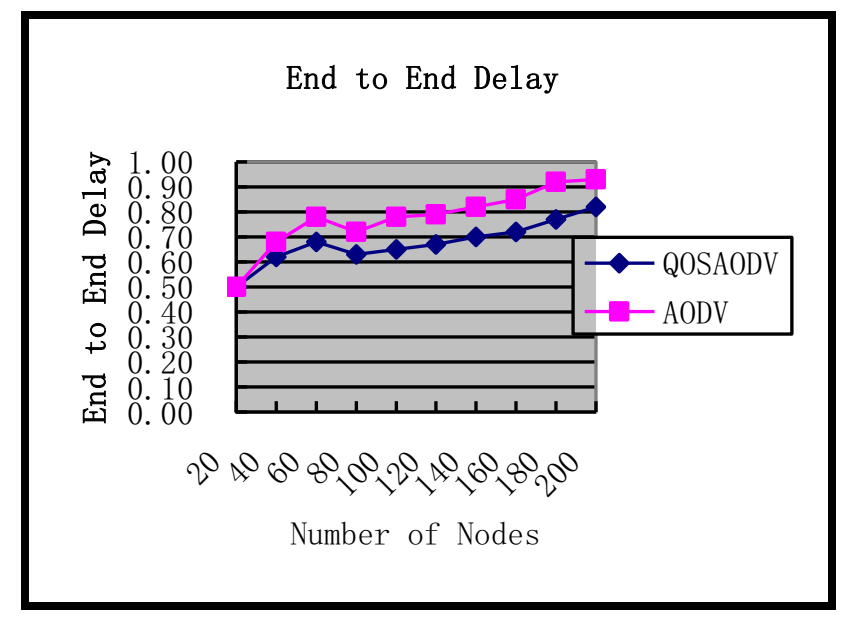

Figure 3.2 end-to-end delay

Figure 3.2 said QOSAODV routing protocol of the average end-to-end delay slightly greater than AODV, because QOSAODV routing protocols to choose the least path is not necessarily the hop. The index compared with packet loss rate and the number of routing chain scission, 
for users is acceptable. Also indicated in the Figure 3.2 QOSAODV routing protocol because of increased HELLO information exchange mechanism and the active routing update mechanism regularly, so in theory is bound to increase the routing overhead. But the main communication ability for on-board wireless ad-hoc network bottleneck is not network physical channel capacity, but within the prescribed period of time how to find the path of the routing to maintain longer greater bandwidth, so add some routing overhead will not affect the larger network of physical communication ability.

\section{Result}

By simulation results, this paper puts forward the correctness of the theory for analyzing the stability of the link, proved QOSADOV routing protocol is better than AODV routing protocol is more suitable for mobile, nodes densely[8] vehicular ad hoc network environment. QOSADOV routing protocol can effectively reduce the average packet loss rate and the routing number of broken chain, realize the vehicular Ad Hoc networks QoS mechanism.

\section{References}

1. Passmann C, Brenzel C, Meschenmoser R. Wireless vehicle to vehicle warning system [J]. SAE2000 World Congress, USA, 2009.

2.Luisa A, Michele P. Inter-vehicle communication and cooperative system: local dynamic safety information distributed among the infra-structure and the vehicles as virtual sensors to enhance road safety [C]. Vehicular Technology Conference, Berlin, 2012.

3. Schmitz R, Leiggener A, Festag A. Analysis of path charac- teristics and transport protocol design in vehicular Ad Hoc networks [J]. Proc of the 63rd IEEE Semiannual Vehicular Technology Conf on VTC-Spring. Melbourne, Australia, 2006.

4. Rudack M, Meincke M, Lott M. On the dynamics of Ad Hoc networks for inter vehicle communication (IVC) $[\mathrm{J}]$. Proceedings of the ICWN 2002, Las Vegas, USA, 2002.

5.Yih-Chun Hu,(2003), Enabling Secure High-Performance Wireless Ad Hoc Networking, PhD Thesis, Carnegie Mellon University (CMU)
6.IIyas M.,(2003), The Handbook Of Wireless Ad Hoc Network, CRC

7.Guoqiang Wang, Damla Turgut,Ladislau Bölöni.Improving routing performance through m-limited forwarding in power-constrained wirless ad hoc networks.ScienceDirect. 2008(68):501-514

8.Sanjay Kumar Dhurandher,Sudip Misra, Mohammad S. Obaidat. EEAODR:An energy-efficient ad hoc on-demand routing protocol for mobile ad hoc networks. INTERNATIONAL JOURNALOF COMMUNICATION SYSTEMS. 2009(22):789-817 